\title{
Exome Sequencing of a Family with Bardet-Biedl Syndrome Identifies the Common Russian Mutation c.1967_1968delTAinsC in BBS7
}

\author{
Evgeny N. Suspitsin ${ }^{a, b} \quad$ Anna P. Sokolenko ${ }^{a, b} \quad$ Lydia V. Lyazina $^{b, c}$ \\ Elena V. Preobrazhenskaya ${ }^{a}$ Alla Y. Lepenchuk ${ }^{b}$ Evgeny N. Imyanitov ${ }^{a}$ b, d \\ ${ }^{a}$ N.N. Petrov Institute of Oncology, ${ }^{\text {b } S t . ~ P e t e r s b u r g ~ P e d i a t r i c ~ M e d i c a l ~ U n i v e r s i t y, ~}{ }^{\mathrm{c} C i t y ~ M e d i c a l ~ G e n e t i c ~ C e n t e r, ~ a n d ~}$ \\ d..I. Mechnikov North-Western Medical University, St. Petersburg, Russia
}

\section{Key Words}

Bardet-Biedl syndrome - BBS7 gene · Ciliopathy - Exome sequencing $\cdot$ Founder alleles

\begin{abstract}
Bardet-Biedl syndrome (BBS) is a rare autosomal recessive ciliopathy characterized by obesity, postaxial polydactyly, retinitis pigmentosa, mental retardation, and kidney abnormalities. At least 19 genes have been shown to be associated with $\mathrm{BBS}$, and therefore, genetic testing is highly complicated. We used an Illumina MiSeq platform for whole exome sequencing analysis of a family with strong clinical features of BBS. A homozygous c.1967_1968delTAinsC (p.Leu656fsX673; RefSeq NM_176824.2) mutation in BBS7 was identified in both affected children, while their healthy sibling and the non-consanguineous parents were heterozygous for this allele. Genotyping of 2,832 DNA samples obtained from Russian blood donors revealed 2 additional heterozygous subjects (0.07\%) with the c.1967_1968delTAinsC mutation. These findings may facilitate the genetic diagnosis for Slavic BBS patients.
\end{abstract}

(c) 2015 S. Karger AG, Basel
Bardet-Biedl syndrome (BBS; OMIM 209900) is a rare genetic ciliopathy, manifesting with blindness-causing retinal dystrophy, postaxial polydactyly, obesity, renal dysfunction, hypogonadism, cognitive abnormalities, and some other severe defects. Molecular diagnosis of this disorder is highly complicated, because mutations in at least 19 genes (BBS1-BBS19) may cause the BBS phenotype [Marion et al., 2012; Forsythe and Beales, 2013; Aldahmesh et al., 2014; Scheidecker et al., 2014]. Sequencing of candidate genes is associated with high setup expenses for appropriate assays, requires significant costs and labor input to analyze the multitude of relevant loci, and has a risk of missing causative mutations in yet unknown BBS genes [Marion et al., 2012; Redin et al., 2012; Scheidecker et al., 2014]. The recent invention of whole exome sequencing (WES) has provided a viable alternative for BBS genetic diagnosis [Ajmal et al., 2013]. To our knowledge, BBS patients have not yet been systematically described in Russia or other Slavic countries. Here, we present a Russian family with BBS which was identified via WES.

\section{KARGER 125}

(c) 2015 S. Karger AG, Base

$1661-8769 / 15 / 0062-0096 \$ 39.50 / 0$

E-Mail karger@karger.com

www.karger.com/msy
Evgeny N. Imyanitov

Laboratory of Molecular Oncology

N.N. Petrov Institute of Oncology

Pesochny 2, St. Petersburg 197758 (Russia)

E-Mail evgeny@imyanitov.spb.ru 


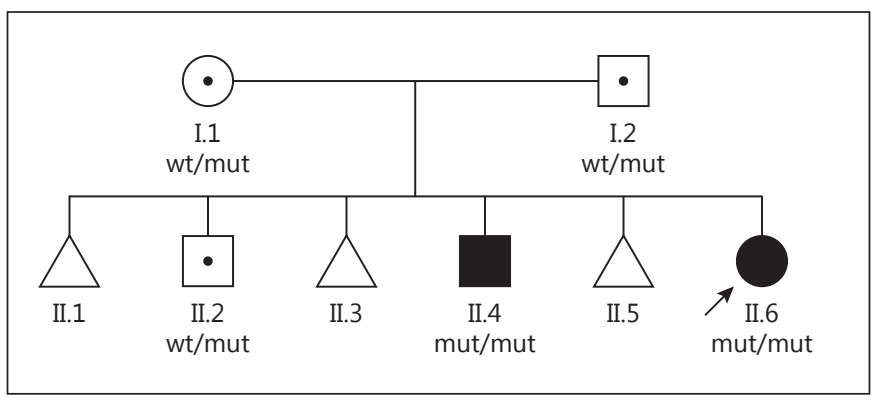

Fig. 1. Russian family with BBS. wt/mut = Heterozygous carrier of the BBS7 c.1967_1968delTAinsC mutation; mut/mut = homozygous carrier of this mutation. The pedigree was drawn using Pedigree Chart Designer (CeGaT, Germany).

\section{Case Report}

A family with 2 cases of presumable BBS requested genetic counseling at the St. Petersburg City Medical Genetic Center, Russia (fig. 1). The parents, being clearly non-consanguineous, were born in distinct regions of Russia (Bryansk and Volgograd). The 35-year-old mother (I.1) reported chronic anemia and mild gastrointestinal dysfunction, while the 41-year-old father (I.2) was healthy. Both parents did not have prior marriages. By the time of the referral, 6 pregnancies had occurred within this family. The first (II.1), the third (II.3), and the fifth (II.5) pregnancy was terminated spontaneously at 6-8 weeks for unknown reasons. The second pregnancy (II.2) resulted in the birth of an apparently healthy boy who is now 16 years old. Unfortunately, children from the fourth (II.4, 7-year-old boy) and the sixth (II.6, 1.5-year-old girl) pregnancy demonstrated characteristic clinical features of BBS.

BBS signs in the affected boy included macrosomy (height: 145 $\mathrm{cm}$; weight: $52 \mathrm{~kg}$; head circumference: $54 \mathrm{~cm}$; chest circumference: $85 \mathrm{~cm}$ ), abdominal obesity, retinitis pigmentosa, history of surgically corrected postaxial polydactyly, prominent forehead, flat nasal bridge, micropenis, mild mental retardation, and a decreased emotional responsiveness. The affected girl had a similar phenotype, including macrosomy (height: $93 \mathrm{~cm}$; weight: $20 \mathrm{~kg}$; head circumference: $50 \mathrm{~cm}$; chest circumference: $66 \mathrm{~cm}$ ), abdominal obesity, history of surgically corrected postaxial polydactyly, prominent forehead, flat nasal bridge, speech delay, and an obstinate attitude. An ophtalmological examination was not carried out due to resistance of the child.

\section{Materials and Methods}

DNA samples from the affected girl and her healthy father were subjected to WES. Exome enrichment was performed using the Nextera Exome Enrichment Kit (Illumina, USA) which is supposed to cover $37 \mathrm{Mb}$ of coding sequences (214,405 exons; $98.3 \%$ of sequences annotated in RefSeq database) and includes all 19 known $B B S$ genes. Massive parallel sequencing was carried out using Illumina MiSeq and included multiple 150-bp reads with approximately $50 \times$ coverage. Sequencing depth across coding re- gions of $B B S 1-B B S 19$ did not significantly differ from the average (online suppl. table 1; www.karger.com/doi/10.1159/000371408). The conversion of nucleotide-specific fluorescent signals was done using MiSeq Reporter software. Reads were aligned to the Human Reference Genome (version hg19) by Burrows-Wheeller Aligner. The obtained files were analyzed using GATK (Genome Analysis Tool Kit) software. The identified differences from the reference sequence were annotated using Annovar resource (www.openbioinformatics.org/annovar/).

\section{Results and Discussion}

WES led to the identification of a homozygous c.1967 1968delTAinsC (p.Leu656fsX673; RefSeq NM_176824.2) germline mutation in the BBS7 gene in the analyzed girl. Her affected brother carried this homozygous BBS7 defect as well, while the healthy brother and both parents were heterozygous carriers of the c.1967_1968 delTAinsC allele. This mutation has already been described by Muller et al. [2010] who detected compound BBS7 heterozygosity (c.1967_1968delTAinsC and c.528+1G>A) in a patient with BBS. BBS7 encodes a subunit of the BBSome complex and is essential for proper cilia functioning. $\mathrm{Mu}-$ tations in BBS7 have been revealed in only $2 \%$ of analyzed BBS patients [Forsythe and Beales, 2013].

The presence of an identical mutation in unrelated parents of patients with BBS is not unexpected. Surprisingly, the Slavic population of Russia and neighboring countries is characterized by pronounced founder effects, so a number of genetic diseases in this part of the world are attributed to recurrent alleles [Dörk et al., 2000; Sokolenko et al., 2010; Jurecka et al., 2012]. By allele-specific PCR, we further analyzed a collection of 2,832 DNA samples obtained from Russian healthy blood donors and revealed 2 additional subjects $(0.07 \%)$ heterozygous for the c.1967_1968delTAinsC allele in BBS7.

This study exemplifies the power of WES for the diagnosis of genetically heterogeneous diseases. Importantly, the DNA analysis was performed using a relatively accessible, laboratory-scale next generation sequencing device. The recurrent nature of the identified mutation, c.1967_1968delTAinsC in BBS7, has now to be taken into account while considering a BBS diagnosis in patients of Slavic origin.

\section{Acknowledgement}

This work was supported by the Russian Foundation for Basic Research (grants 13-04-92613, 13-04-01375 and 14-04-00411). 


\section{References}

Ajmal M, Khan MI, Neveling K, Tayyab A, Jaffar $S$, et al: Exome sequencing identifies a novel and a recurrent $B B S 1$ mutation in Pakistani families with Bardet-Biedl syndrome. Mol Vis 19:644-653 (2013).

Aldahmesh MA, Li Y, Alhashem A, Anazi S, Alkuraya H, et al: IFT27, encoding a small GTPase component of IFT particles, is mutated in a consanguineous family with BardetBiedl syndrome. Hum Mol Genet 23:33073315 (2014).

Dörk T, Macek M Jr, Mekus F, Tümmler B, Tzountzouris J, et al: Characterization of a novel 21-kb deletion, CFTRdele2,3 (21 kb), in the CFTR gene: a cystic fibrosis mutation of Slavic origin common in Central and East Europe. Hum Genet 106:259-268 (2000).
Forsythe E, Beales PL: Bardet-Biedl syndrome Eur J Hum Genet 21:8-13 (2013).

-Jurecka A, Piotrowska E, Cimbalistiene L, Gusina $\mathrm{N}$, Sobczyńska A, et al: Molecular analysis of mucopolysaccharidosis type VI in Poland, Belarus, Lithuania and Estonia. Mol Genet Metab 105:237-243 (2012).

Marion V, Stutzmann F, Gérard M, De Melo C, Schaefer E, et al: Exome sequencing identifies mutations in LZTFL1, a BBSome and smoothened trafficking regulator, in a family with Bardet-Biedl syndrome with situs inversus and insertional polydactyly. J Med Genet 49: 317-321 (2012).

Muller J, Stoetzel C, Vincent MC, Leitch CC, Laurier $\mathrm{V}$, et al: Identification of 28 novel mutations in the Bardet-Biedl syndrome genes: the burden of private mutations in an extensively heterogeneous disease. Hum Genet 127:583593 (2010).
Redin C, Le Gras S, Mhamdi O, Geoffroy V, Stoetzel C, et al: Targeted high-throughput sequencing for diagnosis of genetically heterogeneous diseases: efficient mutation detection in Bardet-Biedl and Alström syndromes. J Med Genet 49:502-512 (2012).

Scheidecker S, Etard C, Pierce NW, Geoffroy V, Schaefer E, et al: Exome sequencing of BardetBiedl syndrome patient identifies a null mutation in the BBSome subunit BBIP1 (BBS18). J Med Genet 51:132-136 (2014).

Sokolenko AP, Iyevleva AG, Mitiushkina NV, Suspitsin EN, Preobrazhenskaya EV, et al: Hereditary breast-ovarian cancer syndrome in Russia. Acta Naturae 2:31-35 (2010).
Suspitsin/Sokolenko/Lyazina/ Preobrazhenskaya/Lepenchuk/Imyanitov 